\title{
Low Readings
}

National Cancer Institute

\section{Source}

National Cancer Institute. Low Readings. NCI Thesaurus. Code C63046.

Reading provided by the device is too low or lower than expected. 